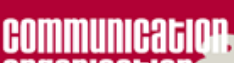
Oryanllatín.

\section{Communication et organisation}

Revue scientifique francophone en Communication organisationnelle

$52 \mid 2017$

Communauté, sociabilité et bien commun: approche internationale

\title{
La communauté en tension : critique de la transparence
}

The community in tension: criticism of transparency La comunidad en tensión: crítica de la transparencia A comunidade em tensão: crítica da transparência

Maria Lucília Marcos

\section{OpenEdition}

1 Journals

\section{Édition électronique}

URL : https://journals.openedition.org/communicationorganisation/5715

DOI : 10.4000/communicationorganisation.5715

ISBN : 979-10-300-0257-7

ISSN : $1775-3546$

Éditeur

Presses universitaires de Bordeaux

Édition imprimée

Date de publication : 1 décembre 2017

Pagination : 113-126

ISBN : 979-10-300-0163-1

ISSN : 1168-5549

\section{Référence électronique}

Maria Lucília Marcos, «La communauté en tension : critique de la transparence », Communication et organisation [En ligne], 52 | 2017, mis en ligne le 01 décembre 2020, consulté le 01 janvier 2022. URL http://journals.openedition.org/communicationorganisation/5715; DOI : https://doi.org/10.4000/ communicationorganisation. 5715 


\title{
La communauté en tension: critique de la transparence
}

\author{
Maria Lucília Marcos ${ }^{1}$
}

\section{La tension au sens des sciences physiques}

Le thème de ce texte porte sur la notion de tension, entendue comme un concept opératoire pour penser la communication dans les organisations humaines ou, plus radicalement, dans l'expérience humaine. Mais avant d'aborder les questions centrées sur des problématiques d'organisation et de communication, faisons tout d'abord un détour par les sciences physiques, où ce terme/concept est récurrent ${ }^{2}$.

La tension physique est l'effort interne de résistance d'un corps soumis à un effort extérieur. En mécanique, dans un corps matériel ou dans un milieu continu soumis à des forces, la mesure de la force interne par unité de surface est appelée tension mécanique. Ces forces internes sont des forces de réaction contre les forces extérieures appliquées au corps.

La tension de surface est un effet physique qui se produit sur la couche de surface d'un liquide qui réagit alors comme une membrane élastique. Les molécules à l'intérieur d'un liquide sont attirées dans toutes les directions par les molécules voisines et, par conséquent, la résultante des forces qui agissent sur chaque molécule est pratiquement nulle. Cette force vers le côté et vers le bas crée la tension sur la surface, ce qui implique qu'elle se comporte comme une fine couche élastique ${ }^{3}$.

1 NOVA FCSH, CIC. Digital/FCSH.UNL. Formation en Philosophie et Sciences de la Communication. Maria Lucília Marcos est Professeure Associée Agrégée en Sciences de la Communication. Associate Professor with Habilitation in Communication Sciences.Coordinator of the Master in Communication Sciences ; marialuciliamarcos@gmail.com

2 Cf. Sabine Mainberger, Spannung II, in J. Ritler, K.Grunder (Ed.), Historisches Worterbuch der Philosophie, Basel/Stuttgart, 1971, vol. 9; Cf. Marco Brusotti, Tensão: um conceito para o grande e o pequeno, trad.Rogério Lopes, Dissertatio, 2011.

3 Par exemple: la goutte d'eau qui se forme dans un robinet conserve sa forme en raison de lélasticité de la surface de la goutte. Un autre exemple: en plaçant avec soin une petite aiguille sur la surface de l'eau, on constate quelle peut demeurer sur la surface de la fine couche sans tomber dans le liquide, en dépit du fait dêtre beaucoup plus dense que l'eau. Un autre exemple encore: ce même effet physique permet aux insectes de marcher sur l'eau. Enfin un autre exemple: celui qui permet aussi à des petits objets métalliques, tels que des aiguilles ou des lames, de flotter à la surface de l'eau. 
La tension tangentielle, tension de coup ou tension coupante, est un type de tension produite par des forces appliquées dans des directions semblables et opposées, mais avec des intensités différentes dans le matériel analysé4.

La tension électrique, aussi connue comme différence de potentiel ou de voltage, est la différence de potentiel électrique entre deux points ou la différence d'énergie électrique potentielle par unité de charge électrique entre deux points. La différence de potentiel correspond au travail qui doit s'exercer, par unité de charge, contre un champ électrique pour déplacer n'importe quelle charge, comme, par exemple, la tension dans le circuit alimenté par une batterie. Une différence de potentiel peut représenter à la fois une source d'énergie (force électromotrice) et une énergie perdue ou stockée (chute de tension). Un voltmètre peut être utilisé pour mesurer la différence de potentiel entre deux points dans un système, un point de référence commun étant habituellement la terre. Par analogie, la tension électrique serait la force responsable du mouvement des électrons.

La tension artérielle fait référence à la pression exercée par le sang contre les parois des artères. La pression artérielle, ainsi que celle de l'ensemble du système circulatoire, est généralement un peu au-dessus de la pression atmosphérique, la différence de pression favorisant l'élasticité des artères. Sa valeur, chez l'individu en bonne santé, varie en permanence, en fonction de l'activité physique, du stress ou de l'état émotionnel.

En résumé, le concept de tension recouvre un champ sémantique qui permet d'identifier quelques termes récurrents : corps, matière, effort, force, résistance, réaction, opposition, intensité, différence, potentiel, pression, travail, charge, énergie, activité, mouvement.

\section{Tension et expérience humaine}

Pour en venir à l'expérience humaine, au sens où les corps humains sont soumis à des tensions sensorielles, physiques, chimiques, mécaniques, électriques et, au-delà de celles-ci (communes à tous les êtres vivants), à d'autres types de tension (émotionnelle, sentimentale, affective, intellectuelle, politique, axiologique, culturelle,...), nous parlerons de tensions au sens où la logique est parfois parmenidéenne - selon le modèle de l'être ou ne pas être - une logique binaire avec une formulation exclusive d'annulation (peutêtre prématurée) de l'un des termes. Mais cette dichotomie est loin d'être exclusive.

Chez l'être humain, les vraies tensions ne se réduisent pas à l'opposition oui vs non. Comme a écrit Paul Celan : "Parle - Mais ne sépare pas le Non du Oui. Donne à tes mots aussi le sens: ménage-lui de l'ombre » (Sprich - Doch scheide das Nein nicht vom Ja. Gib deinem Spruch auch den Sinn: gib ibm den Schatten).

4 Un exemple en est l'application de forces parallèles, mais dans des sens opposés, ou la tension typique produite par le coup en ciseaux. 
Dans l'expérience humaine, les vraies tensions résultent de l'altérité, la contingence, l'indécidable, d'une logique de la préférence.... Préférer c'est suspendre ou interrompre un processus (selon la formule de Bartleby de Melville: I would prefer not to). Les tensions véritables sont irréductibles, persistantes... Ce qui signifie, en référence au champ sémantique de la Physique, qu'elles se maintiennent dans l'effort, la force, la résistance, la réaction, l'opposition, l'intensité, la différence, le potentiel, la pression, le travail, la charge, l'énergie, l'activité, le mouvement... Et qui, en plus du corps et de la matière, sont ressenties dans l'âme. Les vraies tensions se situent dans l'« inter» et, surtout, nous situent dans l'« inter».

Cette expérience se retrouve dans les relations diadiques et communautaires, dans la vie quotidienne de la maison et de la rue, chez le sujet face à lui-même, dans les familles, les écoles, les entreprises, les pays, la diplomatie entre des États, le dialogue inter-culturel, les organisations sociales, en général. La tension définit l'acte politique. L'expérience humaine est, en fait, tensionnelle. C'est incontestablement sa marque.

\section{Société de la transparence}

Pourtant, la « société de la transparence » est devenue aujourd'hui un thème récurrent. Avec l'utilisation généralisée des réseaux numériques, le paradigme de l'information omniprésente, permet de faire l'économie de la profondeur herméneutique de l'événement, de sa dramaturgie, de sa scénographie.

La singularité et l'incommensurable tendent à perdre de leur valeur, remplacés par la valeur nominale de ce qui est comparable et de l'évaluation monétaire. «La société de la transparence est l'enfer de l'égal» (Han 2012 :12). La communication entre égaux et la communication de l'égal uniformisent et stabilisent la négativité et la résistance de l'autre - quand, en fait, l'autre est étrange exactement parce qu'il est autre, indépendamment de considérations qui en cataloguent et en évaluent les différences. Être un autre c'est être autre, en fait et en droit.

Critiquant cette logique de la transparence, Byung-Chul Han (2012) cite Wilhelm von Humboldt qui, déjà au XIX siècle, avait affirmé que la compréhension est toujours incompréhension et que la coïncidence est toujours divergence (p. 13) - la résonance de sens ne se répète jamais, il y aura toujours un plus et un moins de sens qui dérangent la répétition machinale de l'égal (c'est justement le paradoxe de la traduction).

Ceci est d'ailleurs le sens de la honte, du secret, du silence, de l'autonomie, de la distance. Hyperinformation et hypercommunication « témoignent du manque de vérité, voire du manque d'être » (p. 20). La vérité est négativité, elle présuppose l'autre, auquel elle s'oppose. La vérité n'est pas la transparence, elle invite au débat et incite même au combat.

L'idéal de transparence, au lieu d'imposer une vigilance decentrée de la perspective d'un regard despotique (d'après le modèle conçu par Bentham), 
multiplie les regards et les connexions. C'est probablement un gain d'efficacité. Il n'opère pas par l'isolement des individus, il les place plutôt dans un réseau où l'invitation à l'exhibition et au déshabillage est constante. Dans ce panoptique numérique contemporain, les individus se sentent plus libres, ils pensent qu'ils contrôlent la ligne qui sépare ce qu'ils veulent maintenir privé de ce qu'ils veulent exposer et, plus encore, ils estiment que les autres leur renvoient une image d'égale mesure. Il s'agit d'une illusion, comme si la transparence généralisée, dans toutes les directions et dans tous les sens, assurait dans cette réciprocité une confiance partagée et une autonomie croissante. Tout savoir sur tous - voici l'apogée de la transparence, de la confiance, de la liberté.

Le choix de la tension comme concept opératoire pour penser l'expérience humaine dévalue ou démystifie cet idéal de transparence.Dans cette perspective, les individus se font confiance quand, en dépit de ce qu'ils ne savent pas, ils construisent un espace de relations au sein duquel l'attente constitue la seule garantie d'attention à l'autre. L'impératif social de la confiance repose ainsi dans une logique du possible, dans une exigence de loyauté et dans un horizon ouvert à l'imprévu, au contingent et au changement. Tout savoir sur l'autre et lui révéler tout rend « la confiance non nécessaire, remplace la confiance par le contrôle» (Han 2012 : 70). Et tout devient plus fragile et précaire.

Lexcès d'illumination « de soi par soi-même » tend à regrouper les individus autour de marques (brand communitties), en ajoutant de nouvelles vulnérabilités et de nouveaux besoins, en confondant communication et commerce, sans critère extérieur à la consommation, sans mesure, sans négativité du système de profit - des adhésions acritiques, sans condensation ni liberté d'action. Ces " accumulations de je » ne constituent pas de véritables communautés parce que les individus ne sont pas mobilisés par des causes communes, puisqu'ils ne développent pas un espace d'action en dehors du système qui les rassemble.

On peut dire que l'apologie de la transparence est plutôt une question de marketing, en tant que l'expérience demeure irréductiblement ambivalente et que « le sujet retient en soi ses propres contradictions "- en accord avec la définition de Hegel. Mais les mots d'ordre de la visibilité et de la présence dans les réseaux s'imposent comme normalité et comme condition d'appartenance au geistzeit.

Anne Dufourmantelle, psychanalyste et philosophe française, plaide pour un retour à l'intime, en disant que le secret est un luxe :

Nous vivons tous dépliés dans un univers panoptique. On est sans cesse sous le regard de l'autre, du fait de l'évolution des technologies et aussi de cette douce, insidieuse et forte pression du surmoi social. On se sent obligé de tout dire [...]. Le problème de Facebook est le suivant : une idée postée a de la valeur parce qu'elle est choisie par d'autres. C'est le règne implacable du “j'aime parce que tu aimes”, et je suis d'autant plus aimable que cinquante personnes m'aiment et me suivent. Il s'agit donc d'un renforcement du sujet par un groupe qui est une sorte de famille élargie. Et si vous perdez un ami, vous en perdez aussi cinquante d'un coup, comme dans les cours de 
récréation, mais de façon beaucoup plus directe, invasive et brutale. La perfidie du réseau rend l'individu de plus en plus manipulable, performant et identifié $»^{5}$.

\section{Transparence et opacité}

L'exposition publique du sujet, aujourd'hui stimulée par les moyens technologiques, peut atteindre des limites pénibles et illusoires. Freud, à la suite du romantisme allemand, avait montré que le sujet n'est pas transparent à lui-même, dès lors que les actions individuelles dépendent de forces et de motifs inconscients, invalidant l'idée d'une maîtrise de soi absolue. À cette critique psychologique du sujet, Wittgenstein et Saussure ont ajouté une autre critique centrée sur la philosophie du langage: un système préalable de significations linguistiques détermine les discours individuels et enlève à chacun la possibilité de constituer, de son exclusive initiative, le sens de ce qu'il dit. Ces deux approches (fondées en des raisons empiriques et théoriques) sont déterminantes de la pensée du siècle dernier et ébranlent l'autonomie présumée et l'autorité souveraine du sujet.

Toutefois, devant ce scénario de crise, il convient de ne pas renoncer à penser le sujet, au risque d'une perte aliénante. Nous suivons l'étude du philosophe allemand Axel Honneth en faisant valoir que ces forces contingentes (de l'inconscient et du langage) sont des conditions constitutives et des formes d'organisation de l'individualisation du sujet, et non pas des forces étranges, opposées et exorcisables.

Prenant racine dans la pensée de Kant, Honneth (1995) distingue trois sens dans le concept d'autonomie (p. 264) :

1. dans le contexte de la philosophie morale, les jugements moraux autonomes s'orientent selon des principes rationnels, indépendamment des inclinaisons personnelles, selon un régime d'impartialité, indépendamment des causalités empiriques;

2. dans le contexte de la jurisprudence, l'autonomie signifie le droit à l'autodétermination exprimé par des droits légaux garantis à des individus responsables;

3. dans le contexte de la philosophie normative, l'autonomie renvoie à la capacité des sujets à déterminer librement leur vie, sans contraintes, en fonction de critères ou de degrés de maturité psychique, compte tenu de leurs inclinaisons et besoins individuels.

Les deux premiers concepts d'autonomie n'entrent pas dans ce débat : le premier se concentre sur les propriétés des jugements moraux, et non pas sur les personnes concrètes (le problème se pose dans le cas de la projection de l'idéal normatif dans la réalité empirique). Le second concept étend à tout le monde les mêmes droits individuels. Le troisième concept d'autonomie présuppose que chacun a une conscience claire de ses besoins et de la

5 À propos de son dernier essai Défense du secret, Payot, 2015 (Le Figaro.fr). 
connaissance du sens de ses actions, de manière à être en mesure d'organiser librement sa vie. Conscience claire implique transparence et connaissance spécifique du sens implique intentionnalité- demandes excessives que les humains ne possèdent ni ne maîtrisent, en fonction de la présence constante et écrasante de l'inconscient et de la présence d'un système préalable de significations projeté dans les productions discursives.

Penser à nouveaux frais l'autonomie individuelle appelle un décentrement du sujet, au sens saussurien et freudien. Honneth (1995: 266) reprend la psychologie sociale de Mead et la psychanalyse de Winnicott pour montrer que les pouvoirs incontrôlables du langage et de l'inconscient ne limitent pas, mais sont plutôt des conditions d'acquisition de l'autonomie personnelle, en valorisant de façon décisive une théorie de l'intersubjectivité qui puisse réviser les principes classiques des philosophies de la conscience. «I consider the development of a concept of the subject based on a theory of intersubjectivity to be the most promising path for such a position, that is, one which attempts to adapt the idea of individual autonomy to the limiting conditions of the unconscious and language» (p. 263).

De cette analyse ressortent trois niveaux indissociables d'un nouveau modèle de personnalité autonome: l'objectif de transparence est remplacé par la capacité d'articuler de manière créative les besoins et les impulsions intimes, face à soi-même et dans un langage intersubjectif, tout en sachant que la linguistification de l'inconscient sera toujours incomplète; l'objectif de consistance biographique est remplacé par la notion de cohérence narrative de la vie, dans laquelle les différentes étapes de la vie sont des liens dans une chaîne qui peut être conçue de façon rétrospective, selon une "synthèse de l'hétérogène " (Ricœur 1990); l’objectif d'orientation par des principes est remplacé par une sensibilité morale au contexte et au fait que les autres sont confrontés à des contingences.

Dans cette perspective, l'expérience de la reconnaissance est décisive pour la conquête de l'autonomie subjective, tout en suivant un modèle intersubjectif. Et rien dans ce processus n'est transparent, tout est tensionnel. Dans chacun et entre soi-même et l'autre.

\section{Herméneutique narrative}

Cette version de la théorie critique peut configurer ainsi une orientation herméneutique et narrative. "Raconter des histoires est aussi basique que manger - les histoires font notre condition humaine ", écrit Richard Kearney (2002) au début de On Stories (p. 3). Raconter des histoires nous offre un monde partageable, le premier signe de construction d'une communauté. En fait, dans le passage de la nature (physis) à l'Histoire, l'homme a toujours eu besoin de médiations et d'une série de dispositifs: des gestes, des dessins, des mots, des histoires, des images, des outils, des dieux, des croyances, des rituels, 
de l'art, de la technique, des concepts. Et aussi des guerres et des lois. Et c'est cela que nous appelons Histoire.

Kearney s'inspire de Socrate pour affirmer que nous comprenons la vie que nous vivons seulement à travers les histoires que nous raccontons, et qu'une vie qui n'est pas raccontée ne vaut pas la peine d'être vécue (p. 14). Vie en termes individuels, la vie de chacun - laquelle est toujours aussi une vie et un temps avec les autres, même si elle est marquée par les absences. Mais aussi en termes d'expérience collective avec des résonances et des contingences individuelles. Tensionnellemente et singulièrement.

Chaque vie humaine est toujours une histoire implicite, marquée par la finitude d'un principe et d'une fin de l'histoire, affirme Kearney (p.129), s'inspirant du modèle narratif de la Poétique d'Aristote. Le récit ponctue et enchaîne l'existence temporelle - de la bios à la praxis, la vie humaine a tendance à s'interpréter elle-même, en se ré-créant, dans un processus d'invention qui à la fois découvre et crée « ce qui est déjà à la lumière de ce qui n'est pas encore (potentiellement)»(p.132). Entre la contingence de la vie humaine et les histoires racontées s'introduit un intervalle d'extrapolations et de potentialités, dans un processus laborieux de mimésis ou de figuration.

Dans Poétique du Possible - Phénoménologie Herméneutique de la Figuration (1984), livre dédié à Paul Ricœur, Kearney avait élaboré une conception de la créativité qui ne se réduit pas à l'imagination, mais qui se développe dans tous nos actes intentionnels (de perception, de signification, de pratiques). Ce concept est appelé figuration et doit se situer en dehors des dualismes traditionnels de l' " être et ne pas être ", de "présence et absence ", de "réel et imaginaire », en vue de la construction d'une « herméneutique du posible». Cette herméneutique aurait deux sens : «le possible comme dévoilement de l'horizon fini de notre monde vécu - de ce qui est », et « le possible comme horizon transcendant du monde à venir - de ce que doit être " (p.11). Ontologie et éthique pourraient alors se rencontrer, comme dernière tâche de cette étude de Kearney, dans une rélation poétique.

Dans le cadre de ces questions, on retiendra le terme figuration pour montrer que la créativité de l'homme s'exprime dans tous les domaines. En utilisant une méthode phénoménologique, en essayant d'éviter les préjugés métaphysiques onto-théologiques des dualismes mentionnés et qui rendraient obscure la notion de créativité, aussi bien qu'ils gêneraient la description de l'expérience, Kearney soutient la thèse suivant laquelle l'existence humaine est toujours figuration, est toujours transcendantale, ex-tatique, par le dépassement temporel du donné actuel, en vue d'un horizon possible. Nous figurons notre monde comme ceci ou cela: « nous voyons-comment », « nous signifions-comment », " nous imaginons-comment ». Le sens fondamental (s'il existe) n'est jamais présent, mais possible, reste comme une possibilité absente. Laissons ici suspendue la lecture heideggerienne de ce renvoi à un sens absent - fondamental, pour Heidegger, à tous les horizons possibles dans 
le monde, comme sens ultime de la mort. Sens qui unifie toute l'existence et réalise un cercle herméneutique dans lequel la structure "comme si » (als) est avant tout une structure "pré » (vor). Autrement dit n'importe quelle figuration « comme-si » est toujours " pré-figuration » (als-vor) temporisante de son sens possible.

Oui, il importe ici de percevoir que les actes figuratifs primaires - dans les trois modalités de la perception, de l'image et de la signification - enchaînent toujours des figurations et des configurations, un jeu entre des interprétants, une "sémiose illimitée », tel que Peirce nous l'a enseignée. Ce processus métaphorique et métonymique dérive également de la dimension relationnelle du langage qui s'exprime dans la transfiguration du même en altérité possible. Regarder, c'est voir au-delà de ce qu'on voit, parce que l'activité d'identification et de reconnaissance fait appel aux catégories précédentes et se projette en des noms et des expressions différentes; en donnant l'impression de répétition, il s'agit, en fait, de différenciation. Imaginer, c'est construire des images, plutôt que copier des modèles, toute copie étant production d'un autre que soi-même. Signifier, c'est dédoubler le verbe « être » en « être et ne pas être », parce que l'affirmation métaphorique " être comme » prédique tensionnellement, fait « être » et « ne pas être » à la fois. Le terme figuration, présent dans le modèle herméneutique « comme si », permet de comprendre les différentes modalités de représentation - de discours de fiction et de discours sur la réalité - sans l'inconvénient de confondre des plans qui, en fait, se distinguent. Par exemple, la loi de la gravité, théorisée par les physiciens, n'est pas sur le même plan qu'un conte de fées. De même, les personnages de l'histoire sociale et politique ne relèvent pas de fictions identiques à celles qui sont représentées par les contes populaires ou par des romans littéraires.

Le caractère figuratif de la connaissance découle du caractère figuratif du langage. La transparence et l'opacité voilant et dévoilant, le langage n'est jamais littéral, il est toujours figuration. Comme dans les statues grecques de l'époque classique, en particulier dans la représentation du corps féminin, dans laquelle les tuniques couvrent le corps tout en suggérant la position, la forme et le volume des épaules, des seins, de l'abdomen, des fesses, des genoux. Les voiles masquent et montrent, simultanément. Et cette suggestion de nudité déguisée est un effet extraordinaire d'un travail fait sur la pierre, matériau opaque, en faisant de la transparence un effet de sens, une fiction. Si on levait le voile qui nous semble transparent, il ne resterait rien, il n'y aurait rien à voir.

Le caractère figuratif de la connaissance est, en fait, multi-relationnel. Parce qu'il établit des connexions et explique les faits et phénomènes sur la base de ces connexions, parce qu'il relie les explications à d'autres explications, parce qu'il construit des réseaux de concepts qu'on appelle sciences ou philosophies, des réseaux de croyances qu'on appelle mythes, des réseaux entre le profane et le sacré qu'on appelle religions, des «synthèses de l'hétérogène» (une expression de Ricœur) que nous appelons récits. Mais la connaissance est 
encore relationnelle parce que cette expérience n'est possible que lorsque les sujets interagissent entre eux, quand ils mettent en circulation des énoncés en des différentes modalités d'énonciation (de doute, de certitude, de presque-certitude, de compromis, d'adhésion, de refus,...). Et elle est encore relationnelle car cette expérience est essentielle comme lien au monde (le Dasein ou le Mitsein de Heidegger), c'est même une condition de possibilité pour que l'homme ait du monde une expérience dont l'animal est exclu'.

«Raconter des histoires [storytelling] est aussi légal (lawful) et vital que manger» (Kearney 2002: 150). La condition discursive du récit permet le partage d'un monde commun - partage de voix, de désirs, d'intentions, en agencement interactif, selon un modèle de récit de la subjectivité. Citant Habermas, Kearney met en valeur l'intérêt communicationnel du récit d'histoires. Citant Richard Rorty, Kearney souligne les mérites d'une société inspirée par l'imagination narrative davantage que par des sermons ou des traités (1) «Raconter des histoires [storytelling] est aussi légal (lawful) et vital que manger » (Kearney $2002: 150$ ).

La condition discursive du récit permet le partage d'un monde commun - partage de voix, de désirs, d'intentions, en agencement interactif propice au récit de soi. Citant Habermas, Kearney met en valeur l'intérêt communicationnel du récit. Citant Richard Rorty, il souligne les mérites d'une société portée par l'imagination narrative plutôt que par des sermons ou des traités. Dans le récit, c'est le nom propre, l'expérience de vie, la biographie qui prévalent et non les stéréotypes sociaux. On parlera de " Maria », "Louis », ... et non pas des «noirs «, des «femmes», des «gitans ou des «musulmans».

En fait, "The wisdom of the novel comes from having a question for everything...." - nous dit Milan Kundera dans The Book of Laughter and Forgetting ${ }^{7}$. Racontemoi une histoire» - et des images s'affichent dans les grottes ${ }^{8}$. «Raconte-moi une histoire» - et le Génesis de répondre avec le récit de la Tour de Babel. «Raconte-moi une histoire» - et Socrate de déguiser l'embarras érotique avec une légende à propos de l'écriture ${ }^{9}$. «Raconte-moi une histoire» - et Les Filles ${ }^{10}$ chassaient la peur de l'intérieur des maisons.

\section{Project}

Dans une perspective philosophique et politique, Kearney dirige le Guestbook Project ${ }^{11}$ qui encourage l'échange d'histoires entre les membres

\footnotetext{
6 L'Ouvert. De l'homme et de l'animal, Giorgio Agamben, 2002, Paris, Payot et Rivages ; The Strange Order of Things: Life, Feeling, and the Making of Cultures, António Damásio, 2017, Kindle Editions.

7 1999, New York, Perennial Classics ed.

8 Homo Spectator, Marie-José Mondzain, 2007, Paris, Bayard ; Cave of Forgotten Dreams, 2010, 3D documentary film by Werner Herzog (about the Chauvet Cave in southern France, which contains the oldest human-painted images yet discovered. Some of them were crafted around 32,000 years ago).

9 Phèdre, de Platon.

10 As Meninas (Les Filles) - Peintures de Paula Rego

$11 \mathrm{http}: / /$ guestbookproject.org
} 
de groupes en conflit, visant à la réconciliation à travers l'utilisation de l'imagination créatrice. Les termes " hostilité » et " hospitalité » ont la même racine étymologique (hostis) - qui, en tant que réalités, sont en conflit. Dans le cadre du Guestbook Project, les gens sont motivés à reconnaître leurs « traumas transgénérationnels » et à en reconnaître l'impact sur leurs conflits actuels, à travers un processus de storytelling - en racontant et en écoutant des histoires d'inimitié, dans un échange mutuel, afin de susciter une nouvelle histoire, en convertissant l'hostilité en empathie (Exchanging stories, changing history), une éventuelle nouvelle histoire construite sur la reconnaissance d'anciennes divisions, où les deux protagonistes commencent par se reconnaître hostiles puis hôtes (guests). This second crucial move towards a shared, co-created narrative involves a leap of imagination. A radical belief in the impossible (Kearney, in Marcelo 2017 : 11).

Ce projet est fondé sur l'idée que c'est seulement en revisitant les cycles transgénérationaux de répétitions compulsives et de situations non résolues qu'il est possible de réécrire les cicatrices écrites dans le corps et dans la mémoire de chacun, en engendrant de nouvelles lectures de la vie. L'idée d'une communauté préalable est irréaliste. L'idée d'expériences transparentes est illusoire. La reconnaissance de la pluralité et de la différence est la condition de la réconciliation.

On peut faire quelques références concrètes à l'actualité politique ou cosmopolitique, comme dirait Ulrich Beck, selon qui: Le cosmopolitisme est la prise de conscience du destin commun qui lie désormais toutes les parties du monde dans le partage des mêmes risques (2006). C'est pourquoi on doit prendre en considération la dimension transnationale des phénomènes et abandonner le nationalisme méthodologique, car dans un monde globalisé où les réseaux, les discussions et les médias de masse nous touchent tous, les événements nationaux ou locaux concernent de plus en plus l'ensemble de la planète.

Pour Beck, la cosmopolitisation renvoie à un processus multidimensionnel et complexe caractérisé par les interdépendances qui relient en fait les hommes les uns aux autres, bon gré mal gré. Notre vie quotidienne, notre travail, nos rapports amoureux ou d'amitié, les organisations de l'espace public, professionnelles, sportives, culturelles, etc. deviennent cosmopolitiques, transnationales, au sens où elles sont un mélange de différentes origines. La distinction analytique entre nous et les autres est désormais brouillée. Nous faisons partie de la constellation cosmopolite et nous devons en profiter pour construire des institutions cosmopolitiques capables de surmonter les intérêts saisis seulement à l'échelle nationale.

Mais cette approche ne signale pas la fin des différences. En Europe, notamment, il ne s'agit pas de vouloir une société uniforme, mais d'organiser une structure cosmopolite, qui reconnaisse à la fois ses membres dans leurs différences, en visant une combinaison nouvelle de la démocratie nationale et de la démocratie européenne. Il s'agit de penser un scénario transnational pour 
la cosmopolitisation des générations, construit sur de nouveaux concepts, hors des routines et ouvrant d'autres voies. Le modèle cosmopolitique va au-delà d'un modèle universaliste tout court, en mettant l'accent sur la reconnaissance des différences, tant à l'intérieur qu'à l'extérieur du cadre national. Beck parle d'une "polygamie de cultures». "Le cosmopolitisme permet d'exorciser l'idée qu'il faudrait se suicider culturellement pour devenir européen. Il faut cesser de toujours raisonner sur le mode du "ou bien... ou bien" pour saisir les pluralités d'appartenance» (Beck 2006).

Lecadreherméneutique et narratifsuppose unelogique communicationnelle, vraiment interactionnelle, des processus qui déclenchent des récits où les pluralités et les différences peuvent se combiner autrement en créant une nouvelle histoire partagée.

Hospitality is always a wrestle with hostility (Kearney, in Marcelo 2017, p. 10) - dans le cadre du Guestbook Project, cette lutte impose aux protagonistes un complexe processus herméneutique d'échange et d'invention : en Irlande $\mathrm{du}$ Nord, une jeune catholique et une jeune protestante changent d'uniformes et d'écoles pour expérimenter différemment leurs vies et ouvrir une troisième histoire (In Peace, Apart); un Congolais et une Rwandaise tombent amoureux, en dépit de la séparation sociale et nationale (Because I Love Her ); une Palestinienne et une Israélienne portent des vêtements qui sont typiques de l'autre côté (The Other Side) ; un mur divise une famille irlandaise : d'un côté, il y a ceux qui voyagent, de l'autre ceux qui ont un domicile fixe (Hardened Concrete) ; un étudiant arménien de Droit et un académicien turc parlent de leurs identités nationales et perspectives sur le Génocide (Armenian Genocide: An Exchange of Narratives ); des étudiants israéliens et palestiniens se rencontrent pour discuter des identités nationales à Jérusalem (Jerusalem: Across the Divide ); dans une ville ethniquement et linguistiquement divisée, qui est l'invité et qui est l'hôte? (War Letters: To Kill or To Kiss) ; un étudiant japonais et un autre coréen se disputent sur les îlots dans la mer du Japon (Dokdo or Takeshima: Between Islands) ; dans d'autres vidéos, à Mitrovica, Jérusalem, Ankara, Saint Paul, nous voyons des gens développant la même idée - s'il est possible d'échanger des identités dans l'imagination, alors nous croyons qu'il sera possible d'en faire autant dans la vie réelle.

Une solution politique, constitutionnelle, légale ou économique n'est pas possible, affirme Kearney, sans une solution poétique. You need imagination as well as law, narrative as well as a constitution. Or, as some people would put it, faith in the impossible (p. 12).

La reconnaissance des multiples blessures subies au cours des années et des siècles est une tâche urgente qui peut bénéficier, moyennant la puissance thérapeutique des histoires, du storytelling, sans réprimer les différences authentiques ni imposer une conception névrotique de la souveraineté. Ce projet vise à constituer le commun comme lien de singularités, de séparations, d'opacités. Tensionnellement. 


\section{BIBLIOGRAPHIE}

AGAMBEN Giorgio, 2002, L'Ouvert. De l'homme et de l'animal, Paris, Payot et Rivages.

BECK Ulrich, 2006, Revue Sciences Humaines, n 176.

DAMÁSIO António, 2017, The Strange Order of Things: Life, Feeling, and the Making of Cultures, Kindle Editions.

DUFOURMANTELLE Anne, 2015, Défense du Secret, Paris, Payot.

Han Byung-Chul, 2014, A Sociedade da Transparência, Lisboa, Relógio d'Água, 2014 (Transparencezgesellschaft, 2012).

HERZOG Werner, 2010, Cave of Forgotten Dreams, 3D documentary film.

HONNETH Axel, 1995, The Fragmented World of the Social, NY, New York Press.

KEARNEY Richard, 1984, Poétique du Possible - Phénoménologie Herméneutique de la Figuration Paris, Beauchesne.

KEARNEY Richard, 2002, On Stories, London, Routledge.

KUNDERA Milan, 1999, The Book of Laughter and Forgetting, New York, Perennial Classics ed.

MARCELO Gonçalo, 2017, "Narratives and recognition in the flesh: an interview with Richard Kearney", in Philosophy and Social Criticism, SAGE.

MONDZAIN Marie-José, 2007, Homo Spectator, Paris, Bayard.

PLATON, Phèdre.

REGO Paula e BESSA-LUÍS Agustina, 2001, As Meninas, Lisboa, SIC.

VOGEL Matthias, 2012, Media of Reason. A Theory of Rationality, Columbia Univ. Press.

http://guestbookproject.org

Résumé : Lidée d'expériences transparentes est illusoire - aussi bien dans les relations diadiques comme communautaires, dans la vie quotidienne de la maison et de la rue, dans le sujet face à lui-même, que dans les familles, les écoles, les entreprises, les pays, la diplomatie entre des États, le dialogue inter-culturel, les organisations sociales en général. L'expérience humaine est, en fait, tensionnelle. C'est certainement sa marque. Donc l'idée d'une communauté préalable est irréaliste.

Ce text développe des arguments vers une approche hérmeneutique et narrative, inspirée dans le Guestbook Project, dirigé par Richard Kearney.

Mots-clés : contingence, expérience, hospitalité, narrative, tension, transparence.

Abstract: The idea of transparent experiences is illusory - both in the dyadic and community relations, in the everyday life at home and street, in the subject vis-a-vis itself, as in families, in schools, in companies, in countries, in diplomacy between States, in inter-cultural dialogue, in social organizations in general. Human experience is indeed tensional. That's certainly its mark. It follows that the idea of a prior community is unrealistic. 
This text develops arguments towards a hermeneutic and narrative approach, inspired by the Guestbook Project directed by Richard Kearney.

Keywords: Contingency, Experience, Hospitality, Narrative, Tension, Transparency.

Resumo : A ideia de experiências transparentes é ilusória - tanto nas relações diádicas como comunitárias, na vida quotidiana da casa e da rua, no sujeito face a si próprio, como nas familias, nas escolas, nas empresas, nos países, na diplomacia entre Estados, no diálogo inter-cultural, nas organizações sociais em geral. A experiência humana é de facto tensional. Essa é a sua marca. Dai decorre que a ideia de uma comunidade préria é irrealista.

Este texto desenvolve argumentos visando uma abordagem hermenêtica e narrativa, inspirada no Guestbook Project, dirigido por Richard Kearney.

Palavras-chave: Contingência, Experiência, Hospitalidade, Narrativa, Tensão, Transparência. 
